\title{
A Future Direction for Treatment of Alzheimer's Disease via the Oxidative Injury Theory
}

\author{
William K. Summers'*, Roy L. Martin', Yimeng Liu'2, Bernice Peña', Gary M. Marsh ${ }^{2}$ \\ 'Alzheimer's Corporation, 6000 Uptown Blvd, Suite 308, Albuquerque, NM 87110, USA \\ 2Department of Biostatistics, Graduate School of Public Health, University of Pittsburgh, University of Pittsburgh, A410 Crabtree Hall, 130 DeSoto St, Pittsburgh,
} PA 15261, USA

Article Info

\section{Article Notes}

Received: August 23, 2018

Accepted: September 10, 2018

\section{*Correspondence:}

Dr. William K. Summers, M.D., Alzheimer's Corporation,6000

Uptown Blvd, Suite 308, Albuquerque, NM 87110, USA;

E-mail: md@wksummers.com.

(C) 2018 Summers WK. This article is distributed under the terms of the Creative Commons Attribution 4.0 International License
The state of Alzheimer's disease (AD) research is in disarray. The last FDA approved treatments for AD were rivastigmine (2000) and memantine (2003) ${ }^{1,2}$. Since 1990 over a billion dollars have been spent in the pursuit of the next treatment of $\mathrm{AD}$, but the results have been fruitless ${ }^{3}$. In January 2018 Pfizer openly announced that it would no longer invest in potential treatments of $\mathrm{AD}$ or Parkinson's disease $\mathrm{e}^{4}$.

Since 2004 we have been cautioning that pursuit of the beta amyloid hypothesis or the tau hypothesis as causal in AD would not yield practical therapies ${ }^{5}$. Simply stated we felt that amyloid plaques and tangles represented "tombstones" that marked the location of dead neurons. True, tombstones can cause death, but its principle function is to mark the location of contained localized CNS inflammation ${ }^{6}$. The proper model for an amyloid plaque is the relationship of Tuberculous to Ghon complexes. The function of beta amyloid is to absorb the heavy metals released locally from neurons undergoing apoptosis ${ }^{7}$.

The Oxidative Injury (Free Radical) Theory of AD observes physiological aging could be viewed as a gradual, inevitable process, at least partially generated through the accumulation of certain oxidative lesions and ultimate Alzheimer's disease. However, the actual theory requires an individual initial insult to the brain and there are many potential initial brain insults ${ }^{5}$.

If $\mathrm{AD}$ is the product of Oxidative Injury from any of a number of causes which led to localized inflammation in the CNS which smolders and moves, like rheumatoid arthritis, to other areas of the brain.

- It would explain why dementia associated with Traumatic Brain Injury under the microscope looks similar to AD.

- It would explain why viral infections, such as herpes virus, are associated with $\mathrm{AD}^{8}$.

- It would also explain the presence of Beta Amyloid.

- It would explain why beta amyloid appears to be an effort by the brain to maintain homeostasis in a zone of neuroinflammation ${ }^{7}$.

- It would allow one to view Amyloid plaques as tombstones that mark where neurons died, not as a cause of the neuronal death.

- It would explain why NSAIDs data and recent aspirin studies delay $\mathrm{AD}^{9}$

A simple single antioxidant would likely not be sufficient to prevent or treat $\mathrm{AD}^{10,11}$. Likewise minimal amounts of vitamins and minerals 
would be insufficient to benefit brain function as manifest by memory. There are numerous subcellular locations for free radical injury to the brain, requiring an adequate number of antioxidants in adequate doses ${ }^{3,11}$.

In our most recent paper, we compared potent 34 component antioxidant blend (Memory reVITALIZER or AntiOx) to the most popular multivitamin for 'normal' human subjects over $65^{12}$. The popular multivitamin (33 subjects) failed to demonstrate any benefit during this 6 months study. AntiOx (30 subjects), in contrast, showed robust benefit in memory capacity of 'normal' seniors over age 65. Memory testing was done at baseline, one month, three months, and 6 months. Two memory testing instruments were used. The 100 item names-learning paired association test $\left(\mathrm{NLT}_{100}\right)$ looked at Declarative Memory putatively located in the right superior temporal lobe and other higher cortical areas. The 20-word-recall test $\left({ }_{20}\right.$ WRT) is an immediate recall test of Working Memory. This task appears to be a test of hippocampal competence.

AntiOx demonstrated highly significant improvement in Declarative Memory by one month which maintained over 6 months. Likewise, the Working Memory significantly improved by one month. However, statistical analysis of "Change Scores" revealed the improvement did more than maintain. It significantly improved over the period of 6 months, suggesting possible neurogenesis in the hippocampus. Indeed several of the 34 components have demonstrated the ability to provoke neuronal growth factors.

The AntiOx formula was awarded a US Patent. This patent lists the "practical use" of AntiOx in the treatment of $\mathrm{AD}$ and other neurocognitive disorders. However, rules of the marketplace are set by the FDA who demand that all health supplements carry the statement "These statements have not been evaluated by the Food and Drug Administration. This product is not intended to diagnose, treat, cure, or prevent any disease". To declare a product is a health supplement, however, allows immediate access to the marketplace. The cost of supplements thus is often less than $\$ 20$ per month and can be purchased without a special license.

Overall spending on prescription medicines in the United States to be $\$ 337$ billion, in $2015^{13}$. Pharmaceuticals are assumed potentially dangerous, until the manufacturer demonstrates it to be "safe and effective" to the FDA. To state a product has practical use in a disease such as Alzheimer's, then, requires up to two billion dollars and typically eight years of work before receiving FDA approval to enter the market. The cost of ethical pharmaceuticals to the consumer is often unaffordable without insurance. The manufacturers of AntiOx looked at the cost of development and the delay in entry into the market, and elected to market the product immediately in 2000 as a health supplement.
There is a third avenue to the market called "Medical Food". It is a far less complex FDA procedure. The very significant drawback to the Medical Food label is that it requires a prescription to obtain. Thus the cost and inconvenience for "Medical Foods" is substantial. The barrier to the market is almost as significant as a pharmaceutical.

The decision to market AntiOx as a health supplement affected the design of the research to support the value of the product. To have made AD patients the subject of the research would have complicated the consent process and pointed toward the development of an expensive less available pharmaceutical. But $\mathrm{AD}$, under The Oxidative Injury (Free Radical) Theory, is a continuum from normal aging seniors to AD victims. The two large studies of AntiOx have used normal community-dwelling seniors as subjects. The first study was a double blinded placebo/ active agent study ${ }^{11}$. The most recent one compared AntiOx to a typical multivitamin ${ }^{12}$.

There are other antioxidant preparations that have shown benefit in Alzheimer's patients ${ }^{14,15}$. Again, because of the continuum of normal to mild cognitive impairment to $\mathrm{AD}$, it is logical to believe that potent antioxidants would be useful in the prevention and treatment of $\mathrm{AD}$, and other neurodegenerative diseases.

Today, there are several possibilities for treating $\mathrm{AD}$, given the Oxidative Injury Hypothesis. Single focus antioxidants are less likely to give positive results. Complex potent antioxidant formulations can prevent free radical damage in numerous intracellular locations. Complex formulas also allow for synergy, such as the glutathioneascorbate cycle, can enhance beneficial effects ${ }^{16}$.

But knowing that apoptosis and focal-diffuse micro inflammation is part of the process opens other avenues to therapy. It is known some medications to promote neurogenesis. This can be further defined and developed. It is known that anti-inflammatory medications, such as Aspirin and NSAIDs, delay $\mathrm{AD}^{9}$. But agents that downmodulate CNS inflammation with better safety can be sought out. Montelukast may be one of these agents ${ }^{17}$. Likewise, there are potent anti-inflammatory agents used in the treatment of rheumatoid arthritis that can be explored for use in the advanced AD. Still another possibility is the vaccination for the prevention of infectious agents that result in dementia such as Herpes viruses or Lyme's disease $^{8,18}$. Hypothermia treatment of Traumatic Brain Injury is another approach to dementia prevention ${ }^{19}$. There are others, but beyond the scope of discussion here.

Today, there is great hope for treatment of Alzheimer's if one views AD like most other major illnesses. Like cancer, the approach involves thoughtful polypharmacy.

First, all sedatives need to be removed or avoided. 


\section{$3(5): 1-3$}

Dementia often has idiosyncratic responses to sedatives and antipsychotics. They become more confused, often ataxic, and subject to falls and fractures. In cases where agitation exists, use propranolol or other means ${ }^{20}$. Second, initiate antioxidant therapies such as the AntiOx mentioned here. Third, initiate drugs that promote neurogenesis. Most common antidepressants will fill this function. Fourth, add a long-term safe anti-inflammatory agent such as montelukast. Finally, in the later stages of $\mathrm{AD}$, add an acetylcholinesterase inhibitor.

In the future, more sophisticated approaches will become available, but aggressive treatments of AD with currently available tools can give surprising benefits.

\section{References}

1. https://www.centerwatch.com/drug-information/fda-approveddrugs/drug/620/exelon-rivastigmine-tartrate

2. https://www.accessdata.fda.gov/drugsatfda_docs/nda/2003/21 487_Namenda.cfm

3. Summers WK. Current \& future treatments of memory complaints and Alzheimer's disease. Therapy: Future Medicine. 2011; 8(5): 481-504.

4. https://www.reuters.com/article/us-pfizer-alzheimers/pfizer-endsresearch-for-new-alzheimers-parkinsons-drugs-idUSKBN1EW0TN

5. Summers WK. Alzheimer's disease, oxidative injury, and cytokines. J Alzheimer's Dis. 2004; 6: 651-657.

6. http://www.foxnews.com/us/2016/12/12/child-playing-incemetery-killed-when-tombstone-falls-on-him.html

7. Nair G, Perry G, Smith MA, et al. NMR studies of zinc, copper, and iron binding to histidine, the principal metal ion complexing site of amyloid-beta peptide. J Alzheimer's Dis. 2010; 20: 57-66.

8. Itzhaki RF, Lathe R. Herpes viruses and Senile Dementia: First population Evidence for a causal link. J Alzheimers Dis. 2018; 64(2): 363-366. DOI:10.3233/jad-180266

9. Patel D, Roy A, Kundu M, et al. Aspirin binds to PPARa to stimulate hippocampal plasticity and protect memory. Proc Natl Acad Sci USA 2018; 115(31):E7408-E7417. DOI:10.1073/pnas.1802021115

10. Kamal CD, Gadal S, Mhatre M, et al. Antioxidants in central nervous system diseases: Preclinical promise and translational challenges . J Alzheimer's Dis. 2008; 15: 473-493.

11. Summers WK, Martin RL, Cunningham M, et al. Complex Antioxidant Blend improves Memory in community-dwelling seniors. Alzheimer's Dis. 2010; 19: 429-439. DOI:10.3233/JAD-2010-1229

12. Summers WK, Martin RL, Liu Y, Peña B, Marsh GM. Complex antioxidants in a randomized single blind study of memory in seniors. Aging Clin Exp Res. 2018; 30(4): 395-405. DOI 10.1007/s40520017-0788-6.

13. https://www.ahip.org/wp-content/uploads / $2016 / 04$ / HighPriceDrugsReport.pdf

14. Chan A, Paskavitz J, Remington R, et al. Efficacy of a vitamin/ nutriceutical formulation for early-stage Alzheimer's disease: a 1-year, open-label pilot study with an 16-month caregiver extension. Am J Alzheimers Dis Other Demen. 2008; 23: 571-585.

15. Carrasco-Gallardo C, Farias GA, Fuentes P, et al. Can nutraceuticals prevent Alzheimer's disease? Potential therapeutic role of a formulation containing shilajit and complex B vitamins. Arch Med Res. 2012; 43: 699-704.

16. Meister A. Minireview: Glutathione-Ascorbic acid antioxidant system in animals. J Biol Chem 1994; 269: 9397-9400.

17. Grinde B, Engdahl B. Prescription database analyses indicates that the asthma medicine montelukast might protect against dementia: a hypothesis to be verified. Immun Ageing. 2017 Aug 31; 14: 20. doi: 10.1186/s12979-017-0102-7.

18. Kristoferitsch W, Aboulenein-Djamshidian F, Jecel J, et al. Secondary dementia due to Lyme neuroborreliosis. Wien Klin Wochenschr 2018; 130: 468-478. doi.org/10.1007/s00508-018-1361-9

19. Chiu AW, Hinson HE. Future Directions for Hypothermia following Severe Traumatic Brian Injury. Semin Respir Crit Care Med. 2017 Dec; 38(6): 768-774. doi: 10.1055/s-0037-1607989.

20. Summers, WK. Use of propranolol in management of agitated demented patients: safety and effectiveness. J Alzheimer's Dis. 2006; 9: $69-75$ 